Proyecciones

Vol. 19, $\mathrm{N}^{\circ}$ 1, pp. 1-17, May 2000

Universidad Católica del Norte

Antofagasta - Chile

\title{
A COMPACTNESS EMBEDDING LEMMA, A PRINCIPLE OF SYMMETRIC CRITICALITY AND APPLICATIONS TO ELLIPTIC PROBLEMS *
}

\author{
DANIEL CARLOS de MORAIS FILHO \\ MARCO A. S. SOUTO \\ Universidade Federal de Paraíba, Campus II - Brasil \\ and \\ JOÃO MARCOS DO O. \\ Universidade Federal de Paraíba, Campus I - Brasil
}

\begin{abstract}
In this paper, we present a compactness embedding theorem of a subspace of a the Sobolev space $W^{1, p}\left(\mathbf{R}^{\mathbf{N}}\right)$ into $L^{s}\left(\mathbf{R}^{\mathbf{N}}\right)$ spaces, formed by functions $u(x, y)$ which are spherically symmetry with respect to first variable and $\int a(z)|u|^{p} d z<\infty$, where $a$ is a continuous function satisfying $a(z)>a_{0}>0$ if $|z| \geq R$, for a large $R>0$ and such that $\lim _{|y| \rightarrow \infty} a(x, y)=+\infty$ uniformly for $x$. Moreover, we give two applications to elliptic problems.
\end{abstract}

1991 Mathematics Subject Classifications: 35A05, 35A15 and 35J20.

Key words and phrases: Compact embeding, critical Sobolev exponents, Palais-Smale condition and mountain-pass theorem, elliptic systems.

${ }^{*}$ The authors are partially sponsored by CNPq/Brazil. 
2 D. C. de Morais Filho, João Marcos do Ó \& M. A. S. Souto

\section{Introduction}

In this article we will prove a compactness embedding theorem of a certain subspace of the Sobolev space $W^{1, p}(\mathbf{R})^{N}$ into $L^{s}(\mathbf{R})^{N}$ spaces, for some $s>0$. These results are very important and appear when one deals with some kind of elliptic problems, two of which we present in Section 4. The obstacle one faces when dealing these specific kind of theorems is the lack of compactness, since we shall work with functions defined in the whole $\mathbf{R})^{N}$ and with the critical Sobolev exponent $p^{*}=$ $N p /(N-p)$ of the immersion $W^{1, p}\left(\mathbf{R}^{N}\right) \hookrightarrow L^{P}\left(\mathbf{R}^{N}\right)$.

In order to state more precisely our result, we consider a nonnegative continuous function $a: \mathbf{R}^{N}=\mathbf{R}^{L} \times \mathbf{R}^{M} \rightarrow \mathbf{R}(L \geq 2)$ satisfying the following assumptions:

$\left(a_{o}\right) a(x, y) \geq a_{o}>0$ if $|(x, y)| \geq R$,for a large $R>0$;

$\left(a_{1}\right) a(x, y) \rightarrow+\infty$ when $|y| \rightarrow+\infty$ uniformly for $x \in \mathbf{R}^{L}$;

$\left(a_{2}\right) a(x, y)=a\left(x^{\prime}, y\right)$ for all $x, x^{\prime} \in \mathbf{R}^{L}$ with $|x|=\left|x^{\prime}\right|$ and all $y \in \mathbf{R}^{M}$.

Let us define the real $W^{1, p}$-subspace

$$
\begin{gathered}
\widetilde{E}=\left\{u \in W^{1, p}\left(\mathbf{R}^{L} \times \mathbf{R}^{M}\right): u(x, y)=u\left(x^{\prime}, y\right), x, x^{\prime} \in \mathbf{R}^{L},|x|=\left|x^{\prime}\right|,\right. \\
\left.y \in \mathbf{R}^{M}\right\} .
\end{gathered}
$$

and the reflexive space

$$
E_{a}=\left\{u \in \widetilde{E}: \int_{\mathbf{R}^{L} \times \mathbf{R}^{M}} a(z)|u|^{p} d z<\infty\right\}, z=(x, y),
$$

endowed with the corresponding norm

$$
\|u\|_{a}^{p}=\int_{\mathbf{R}^{L} \times \mathbf{R}^{M}}|\nabla u|^{p} d z+\int_{\mathbf{R}^{L} \times \mathbf{R}^{M}} a(z)|u|^{p} d z
$$

The main result of this paper is the following

Theorem 1.1. If $\left(a_{0}\right)-\left(a_{2}\right)$ hold then the Banach space $E_{a}$ is continuously immersed in $L^{s}\left(\mathbf{R}^{N}\right)$ if $p \leq s \leq p^{*}$, and compactly immersed if $p<s<p^{*}$. 
Lions in [18], extends a compactness result due to Strauss (see [24]). He showed that if $\Omega \subset \mathbf{R}^{M}$ is a bounded domain and $q \in(p, p N /(N-$ $p)$ ), then the restriction to $W_{0, r}^{1, p}\left(\mathbf{R}^{L} \times \Omega\right)$ of the Sobolev embedding from $W_{0}^{1, p}\left(\mathbf{R}^{L} \times \Omega\right)$ to $L^{q}\left(\mathbf{R}^{L} \times \Omega\right)$ is compact, where $W_{0, r}^{1, p}\left(\mathbf{R}^{L} \times \Omega\right)$ is the closed subspace of $W_{0}^{1, p}\left(\mathbf{R}^{L} \times \Omega\right)$ consisting of functions $u \in W_{0}^{1, p}\left(\mathbf{R}^{L} \times \Omega\right)$ which are spherically symmetric with respect to the first variable.

Recently, Costa in [12] (see also $[13,21]$ ) proved a result like the above theorem under the assumption that the function $a$ is coercive, i.e., $a(z) \rightarrow+\infty$ as $|z| \rightarrow+\infty$. Therefore, Theorem 1.1 complements these compactness results mentioned above.

\section{A compactness embedding result - Proof of The- orem 1}

In what follows $C$ will denote a generic positive constant.

First of all, we are going to prove that if condition $\left(a_{0}\right)$ holds then the Banach space $E_{a}$ is continuously immersed in $L^{s}\left(\mathbf{R}^{N}\right)$ for all $s \in\left[p, p^{*}\right]$. Notice that $\left(a_{0}\right)$ yields that

$$
\begin{gathered}
\int_{\mathbf{R}^{L} \times \mathbf{R}^{M}}|u|^{p} d z \leq \int_{\{|(x, y)| \leq R\}}|u|^{p} d z+ \\
a_{o}^{-1} \int_{\{|(x, y)| \geq R\}} a(z)|u|^{p} d z \leq\left. C|| u\right|_{a} ^{p},
\end{gathered}
$$

where we have used the continuity of the Sobolev embedding for bounded domains. On the other hand, the Sobolev-Gagliardo-Nirenberg inequality asserts that there exists positive constant $S$ such that

$$
\int_{\mathbf{R}^{L} \times \mathbf{R}^{M}}|u|^{p^{*}} d z \leq S \int_{\mathbf{R}^{L} \times \mathbf{R}^{M}}|\nabla u|^{p} d z .
$$

Therefore, from inequalities (2.1) and (2.2) we have the continuity of the embedding for $s=p$ and $s=p^{*}$. The continuity of the immersion for a fixed $s \in\left(p, p^{*}\right)$, follows from the following interpolation inequality

$$
\|u\|_{L^{s}} \leq\|u\|_{L^{p}}^{1-t}\|u\|_{L^{p^{*}}}^{t}
$$

where $0 \leq t \leq 1$ is such that $1 / s=(1-t) / p+t / p^{*}$.

Now, assuming $\left(a_{0}\right)-\left(a_{2}\right)$, we are going to prove the compactness of the embedding of the spaces $E_{a} \hookrightarrow L^{s}\left(\mathbf{R}^{N}\right)$ for $s \in\left(p, p^{*}\right)$. For this 
purpose it suffices to show that for any weakly convergent sequence $u_{k} \rightarrow 0$ in $E_{a}$, as $k \rightarrow \infty$, we have that, up to subsequences, the convergence holds strongly in $L^{s}\left(\mathbf{R}^{L} \times \mathbf{R}^{M}\right)$. Since $\left(a_{1}\right)$ holds we may choose an unbounded increasing sequence of positive real numbers $\left(r_{n}\right)$ such that for all $x \in \mathbf{R}^{L}$ we have $a(x, y)>n$, if $|y| \geq r_{n}$. So,

$$
\begin{gathered}
\int_{\mathbf{R}^{L} \times\{|y| \geq r \mathbf{n}\}}\left|u_{k}\right|^{p} d z \leq \frac{1}{n} \int_{\mathbf{R}^{L} \times\{|y| \geq r \mathbf{n}\}} a(x, y)\left|u_{k}\right|^{p} d z \\
\leq \frac{\|\left. u_{k}\right|_{a} ^{p}}{n} \leq \frac{C}{n},
\end{gathered}
$$

for all natural numbers $k$ and $n$. This last inequality together with the interpolation inequality $(2.3)$ yields that

$$
\int_{\mathbf{R}^{\left.\mathbf{L}_{\times}|y| \geq r \mathbf{n}\right\}}}\left|u_{k}\right|^{s} d z \leq \frac{C}{n^{s(1-t) / p}} .
$$

On other hand, using a result due to Lions [18] ( Lemma IV.2 ), for each natural number $j$ we have a subsequence $\left(u_{k_{j}}\right)$ of $\left(u_{k}\right)$ such that

$$
\int_{\mathbf{R}^{\mathbf{L}} \times\left\{|y| \leq r_{j}\right\}}\left|u_{k_{j}}\right|^{s} d z \leq \frac{1}{j} .
$$

The proof follows by estimates (2.5), (2.6) and a diagonal type argument.

\section{A principle of symmetric criticality}

Now we state a version for reflexive Banach spaces, of the well known principle of symmetric criticality due to Palais [22]. Let $E$ be a reflexive Banach space (endowed with the norm $|\cdot|$ ) such that: "for each $S \in E^{*}$,

(3.1) there exists a unique $u_{o} \in E$ such that $(f u)=|u|^{2}=|S|_{E^{*}}^{2} "$

(here $(\cdot, \cdot)$ denotes the bracket duality in $E^{*} \times E$ and $E^{*}$ denotes the dual space of $E$ ). For example: Hilbert spaces, uniformly convex spaces and Sobolev spaces $W^{1, p}$, with $1<p<+\infty$, satisfy unicity condition (3.1). Suppose that $G$ is a subgroup of isometries $g: E \rightarrow E$. Consider the $G$-invariant closed subspace $\Sigma=\{u \in E: \mathrm{g} u=\mathrm{g}(u)=u$, for all $g \in G\}$. 
Let $f$ be a continuous functional on $E$ such that

$$
(f, u)=0, u \in \Sigma
$$

and $f$ is invariant under the action of $G$, that is, $f \circ g=f$, for all $g \in G$ In this case $\Sigma \subset \operatorname{Ker} f$. We claim that under the above assumptions $f \equiv 0$. On the contrary way, since (3.1) holds, there exists a unique $v_{o} \in E \backslash\{0\}$ such that $\left(f, v_{o}\right)=\left|v_{o}\right|^{2}$ and $|f|_{E^{*}}=\left|v_{o}\right|$. Notice that

$$
\left(f, g v_{o}\right)=\left(f \circ g, v_{o}\right)=\left(f, v_{o}\right)=\left|v_{o}\right|^{2}=\left|g v_{o}\right|^{2}
$$

and $|f|_{E^{*}}=\left|v_{o}\right|=\left|g v_{o}\right|$ for all isometry $g \in G$. By the unicity of $v_{o}$ we have $g v_{o}=v_{o}$ for all $g \in G$ which implies that $v_{o} \in \Sigma \subset \operatorname{Ker} f$, contrary to the fact that $v_{o} \neq 0$.

We are now able to prove the proposition.

Proposición 3.1. Let $E, G$ and $\Sigma$ be as above and $I$ be a $C^{1}$-functional defined on $E$ such that $I \circ g=I$, for all $g \in G$. Then $u \in \Sigma$ is a critical point of $I$ if and only if $u$ is a critical point of $\left.I\right|_{\Sigma}$ (the restriction of the functional $f$ to the set $\Sigma$ ).

Proof. Observe that linear functional $I^{\prime}(u)$ is invariant under the action of $G$. Taking $f=I^{\prime}(u)$, the proof follows from the above remarks .

\section{Application to a $p$-Laplacian equation}

Our purpose in this section is obtaining a solution for the global semilinear elliptic problem:

$$
\left\{\begin{array}{c}
-\Delta_{p} u+a(z) u^{p-1}=\lambda u^{q}+u^{p^{*}-1}, \mathbf{R}^{N} \\
u \geq 0, u \neq 0, \int_{\mathbf{R}^{\mathbf{N}}}|\nabla u|^{p}<\infty .
\end{array}\right.
$$

where $\Delta_{p} u=\operatorname{div}\left(|\nabla u|^{p-2} \nabla u\right), a$ satisfies $\left(a_{o}\right),\left(a_{1}\right),\left(a_{2}\right), p^{*}=N p /(N-$ p) ; $p-1<q<p^{*}-1, \lambda>0$ and $N=L+M>p, L \geq 2$.

In the last years, several researchers have been studying variants

of problem (4.1). Among others, in bounded domains, we can cite 
the pioneering article due to Brezis \& Nirenberg [10] which treats the case $a \equiv 0$ and $p=2$. Also in bounded domains, Azorero \& Alonzo in [4], [5] and [6] generalize some similar results for the $p$-Laplacian operator, and Egnell in [14]generalizes some results of [11]. In the unbounded domains case, we cite Rabinowitz [23] and [12]. Rabinowitz considers a more general non-linearity, however he does not treat the Sobolev critical exponent case. Benci \& Cerami [7] consider the problem (4.1) when $\lambda=0$, and [2] deals with the case where $\lambda$ is replaced by an integrable function.

Theorem 4.1. Suppose that $\lambda>0$ and that $\left(a_{o}\right),\left(a_{1}\right)$ and $\left(a_{2}\right)$ are satisfied. If one of the following inequality holds

(i) $N \geq p^{2}$ and $p-1<q<p^{*}-1$;

(ii) $p<N<p^{2}$ and $q>p^{*}-\frac{p}{p-1}-1$

(ii) $p<N<p^{2}$ and $p-1<q<p^{*}-\frac{p}{p-1}-1$, and large $\lambda$.

Then problem (4.1) possesses a nontrivial classical solution $u \in E_{a}$. Proof. The proof consists in using variational methods to get critical points of the Euler-Lagrange functional associated to (4.1). We follow the same steps made in [19] and [2].

Define on $E_{a}$ the functional:

$$
I(u)=\frac{1}{p}\|u\|_{a}^{p}-\frac{\lambda}{q+1} \int_{\mathbf{R}^{N}}\left(u^{+}\right)^{q+1}-\frac{1}{p^{*}} \int_{\mathbf{R}^{N}}\left(u^{+}\right)^{p^{*}}
$$

where $u^{+}(z)=\max \{u(z), 0\}$ and $u^{-}(x)=\min \{-u(x), 0\}$.

The critical points of $I$ are precisely the weak solutions of (4.1). These solutions may be regularized.

Using Theorem 1.1 one can check that $I$ is a well-defined $C^{1}\left(E_{a}\right)$ functional. It is easy to verify that

$$
\frac{\lambda}{q+1} \int_{\mathbf{R}^{N}}\left(u^{+}\right)^{q+1}+\frac{1}{p^{*}} \int_{\mathbf{R}^{N}}\left(u^{+}\right)^{p^{*}}=o\left(\|u\|_{a}^{p}\right), \text { as } u \rightarrow 0
$$

and hence that $I$ has a local minimum at the origin. This is not a global minimum. Indeed, if $u \in E \backslash\{0\}, u \geq 0$, we have that

$$
I(t u)=\frac{t^{p}}{p}\|u\|_{a}^{p}-\frac{\lambda t^{q+1}}{q+1} \int_{\mathbf{R}^{N}}\left(u^{+}\right)^{q+1}-\frac{t^{p^{*}}}{p^{*}} \int_{\mathbf{R}^{N}}\left(u^{+}\right)^{p^{*}} .
$$


Choosing $u \in E_{a}$ such that $\int_{\mathbf{R}^{\mathbf{N}}}\left(u^{+}\right)^{p^{*}} \neq 0$, we conclude that $I(t u) \rightarrow$ $-\infty$ as $t \rightarrow \infty$.

So, we have just seen that $I$ has the Mountain Pass Theorem Geometry.

Let $e \in E_{a}$ such that $I(e)<0$, and define

$$
\Gamma=\left\{g:[0,1] \rightarrow E_{a}, \text { continuous }: g(0)=0, g(1)=e\right\}
$$

and

$$
c=\inf _{g \in \Gamma} \max _{0 \leq t \leq 1} I(g(t)) .
$$

Thus $c$ is the mountain pass minimax value associated to $I$. Assertion (4.2) implies that $c>0$. At this moment, it is important to notice that $c$ is not the minimax value associated to the Euler Lagrange functional of problem (4.1) defined in the whole $W^{1, p}\left(\mathbf{R}^{N}\right)$.

Using an application of the Ekeland Variational Principle (Theorem 4.3 of [20]) we may prove the Mountain Pass Theorem without $(P S)$ condition, this is, there exists a sequence $\left(u_{m}\right) \subset E_{a}$ such that

$$
I\left(u_{m}\right) \rightarrow c, I^{\prime}\left(u_{m}\right) \rightarrow 0
$$

A standard argument proves that the above sequence $\left(u_{m}\right)$ is bounded.

The following lemma shows that we can choose a vector $e \in E_{a} \backslash\{0\}$ in the definition of $\Gamma$, such that $I(e)<0$ and

$$
0<c<\frac{1}{N} S_{p}^{\frac{N}{p}}
$$

where $S_{p}$ is the best constant of the Sobolev immersion $W^{1, p}\left(\mathbf{R}^{N}\right) \subset L^{p^{*}}\left(\mathbf{R}^{N}\right)$. Precisely,

$$
S_{p}=\inf _{u \in W^{1, p}\left(\mathbf{R}^{N}\right)} \frac{\int_{\mathbf{R}^{N}}|\nabla u|^{p}}{\left(\int_{\mathbf{R}^{N}}|u|^{p^{*}}\right)^{\frac{p}{p^{*}}}} .
$$

Using the above facts and arguments due to Brezis \& Nirenberg [11], we will show that the choice in (4.6) implies in obtaining a non-trivial solution of (4.1). 
8 D. C. de Morais Filho, João Marcos do Ó É M. A. S. Souto

Lemma 4.2. Suppose that $\lambda>0$ and one of the following conditions is satisfied:

(i) $N \geq p^{2}$ and $p-1<q<p^{*}-1$;

(ii) $p<N<p^{2}$ and $q>p^{*}-\frac{p}{p-1}-1$;

(ii) $p<N<p^{2}$ and $p-1<q<p^{*}-\frac{p}{p-1}-1$, and large $\lambda$.

Then, there is a vector $e \in E_{a} \backslash\{0\}, e \geq 0, I(e)<0$ such that

$$
\sup _{t \geq 0} I(t e)<\frac{1}{N} S_{p}^{\frac{N}{p}},
$$

Proof. See [1], page. 56.

In order to complete the proof of Theorem 4.1, let us consider $e \in E_{a} \backslash\{0\}$ given by Lemma 4.2. Let $\left(u_{m}\right)$ be the sequence in $E_{a}$ satisfying (4.5). We may assume that

$$
\begin{gathered}
u_{m} \rightarrow u \text { in } E_{a} \\
u_{m} \rightarrow u \text { in } L^{s}\left(\mathbf{R}^{N}\right), p<s<p^{*} \\
u_{m}(x) \rightarrow u(x) \text { a.e. in } \mathbf{R}^{N} .
\end{gathered}
$$

The above limits yields that $u$ must be a critical point of $I$ in $E_{a}$ (see $[1])$, that is,

$$
I^{\prime}(u)=0 .
$$

We claim that $u \neq 0$. In fact, if $u \equiv 0$ and taking $l \geq 0$ such that

$$
\int_{\mathbf{R}^{N}}\left(\left|\nabla u_{m}\right|^{p}+a\left|u_{m}\right|^{p}\right) \rightarrow l,
$$

then

$$
\int_{\mathbf{R}^{N}}\left(u_{m}^{+}\right)^{p^{*}} \rightarrow l,
$$

since $I^{\prime}\left(u_{m}\right) \rightarrow 0$ and $E \subset L^{q+1}\left(\mathbf{R}^{N}\right)$ compactly. Therefore using the fact that $I\left(u_{m}\right) \rightarrow c$, we get

$$
N c=l .
$$


From the definition of $S_{p}$,

$$
\begin{gathered}
\int_{\mathbf{R}^{N}}\left(\left|\nabla u_{m}\right|^{p}+a\left|u_{m}\right|^{p}\right) \geq \int_{\mathbf{R}^{N}}\left|\nabla u_{m}\right|^{p} \geq S_{p}\left(\int_{\mathbf{R}^{N}}\left|u_{m}\right|^{p^{*}}\right)^{\frac{2}{p^{*}}} \\
\geq S_{p}\left(\int_{\mathbf{R}^{N}}\left(u_{m}^{+}\right)^{p^{*}}\right)^{\frac{2}{p^{*}}} .
\end{gathered}
$$

Passing to the limit in the last inequalities, we achieve that

$$
l \geq S_{p} l^{\frac{2}{p^{*}}}
$$

and by (4.8) that

$$
c \geq \frac{1}{N} S_{p}^{\frac{N}{p}}
$$

which contradicts the above choice of $e$ and thus the claim is proved.

Observe that $I^{\prime}(u) u^{-}=0$ implies that $\int_{\mathbf{R}^{\mathbf{N}}}\left|\nabla u^{-}\right|^{p}+a(x)\left(u^{-}\right)^{p}=0$ and then $u^{-} \equiv 0$. Hence $u \geq 0$.

Notice that up to this moment we do not know if $u$ satisfies (4.1) in the $W^{1, p}\left(\mathbf{R}^{N}\right)$ sense but, thanks the Proposition 3.1 used with

$$
E=\left\{v \in W^{1, p}\left(\mathbf{R}^{N}\right): \int_{\mathbf{R}^{N}} a(z)|v|^{p} d z<\infty\right\},
$$

$G=\left\{g: E \rightarrow E: g(v)=v \circ\left(\begin{array}{cc}R & 0 \\ 0 & I d\end{array}\right)\right.$, where $R$ is a rotation in $\mathbf{R}^{L}$

( $I d$ is the $M \times M$ identity matrix) we have $\Sigma=E_{a}$ and $u$ is a critical point of $I$ in whole $E$. Theorem 1 is proved.

\section{Application to a system of elliptic equations}

Our aim in this section is to apply Theorem 1.1 and Proposition 3.1 to find solutions for the following system of elliptic equations

$$
\left\{\begin{array}{rlr}
-\Delta u+a(z) u & =\lambda \frac{2 \gamma}{\gamma+\delta} u^{\gamma-1} v^{\delta}+\frac{2 \alpha}{2^{*}} u^{\alpha-1} v^{\beta}, \quad \mathbf{R}^{N} \\
-\Delta v+b(z) v & =\lambda \frac{2 \delta}{\gamma+\delta} u^{\gamma} v^{\delta-1}+\frac{2 \beta}{2^{*}} u^{\alpha} v^{\beta-1}, \quad \mathbf{R}^{N} \\
& u, v>0
\end{array}\right.
$$

where $N=L+M \geq 3$ and $L \geq 2$. 
10 D. C. de Morais Filho, João Marcos do Ó Ẻ M. A. S. Souto

Our goal is to demonstrate the theorem.

Theorem 5.1. Suppose that the real functions $a$ and $b$ fulfill assumptions $\left(a_{0}\right),\left(a_{1}\right)$ and $\left(a_{2}\right)$. If the following hypotheses hold

$$
\begin{gathered}
\gamma, \delta>1 \text { and } q=\gamma+\delta<2^{*}, \\
\alpha, \beta>1 \text { and } \alpha+\beta=2^{*}, \\
q>1 \text { and } N \geq 4 \text { or } 3<q<5 \text { and } N=3
\end{gathered}
$$

then for every $\lambda>0$ system (5.1) has a solution.

Moreover, the same results is still valid if (5.4) is replaced by

$$
N=3,1<q \leq 3 \text { and } \lambda>0 \text { is sufficiently large. }
$$

We shall follow the same route of the previous section. For this purpose let us consider the cross product Banach space $E=E_{a} \times E_{b}$ endowed with the norm $\|(u, v)\|_{E}^{2}=\|u\|_{a}^{2}+\|v\|_{b}^{2}$.

The weak solutions of (5.1) are the critical points of the functional

$$
I: E \rightarrow \mathbf{R}
$$

defined by

$$
\begin{gathered}
I(u, v)=\frac{1}{2}\|(u, v)\|_{E}^{2}-\frac{2 \lambda}{\gamma+\delta} \int_{\mathbf{R}^{N}}\left(u^{+}\right)^{\gamma}\left(v^{+}\right)^{\delta}- \\
\frac{2}{2^{*}} \int_{\mathbf{R}^{N}}\left(u^{+}\right)^{\alpha}\left(v^{+}\right)^{\beta}
\end{gathered}
$$

Theorem 1.1 and the following lemma assure that $I$ is a well defined functional, and indeed by a straightforward computation, a $C^{1}-$ functional defined on $E$.

Lemma 5.2. For $\alpha^{\prime}+\beta^{\prime}=r \leq 2^{*}$, there exists a constant $K$ such that

$$
\left(\int_{\mathbf{R}^{\mathbf{N}}}|u|^{\alpha^{\prime}}|v|^{\beta^{\prime}}\right)^{1 / r} \leq K\|(u, v)\|_{E}
$$


for all $(u, v) \in E$.

Proof. The proof results from the constants

$$
S_{a}=\inf _{u \in E a \backslash\{0\}} \frac{\int_{\mathbf{R}^{N}}\left(|\nabla u|^{2}+a(z)|u|^{2}\right) d z}{\left(\int_{\mathbf{R}^{N}}|u|^{r} d z\right)^{2 / r}}
$$

and $S_{b}$, defined likewise the above one, and the inequality

$$
|u|^{\alpha^{\prime}}|v|^{\beta^{\prime}} \leq|u|^{r}+|v|^{r}
$$

Remark 1. Taking $a=b, u=v$ in (5.1) we get the scalar case (4.1) with $p=2$.

Lemma 5.2, hypotheses (5.2), (5.3) and Theorem 1.1 guarantee that the functional $I$ has the Mountain Pass Theorem geometry. Hence, as made in the previous section, we may find a sequence $\left(u_{n}, v_{n}\right) \subset E$ such that

$$
I\left(u_{n}, v_{n}\right) \rightarrow c \text { in } \mathbf{R} \text { and } I^{\prime}\left(u_{n}, v_{n}\right) \rightarrow 0 \text { in } E^{\prime},
$$

where $c$ is defined likewise in (4.3) and (4.4), replacing $E_{a}$ by $E$. The sequence in (5.8) is bounded in $E$.

It is standard to assume and prove that the sequence $\left(u_{n}, v_{n}\right)$ weakly converges to $\left(u_{o}, v_{o}\right) \in E$ and then that we have

$$
I^{\prime}\left(u_{o}, v_{o}\right)=0
$$

i.e, $\left(u_{o}, v_{o}\right)$ is a weak solution for (5.1).

From now on we shall be concentrated in proving that $u_{o}, v_{o}>0$. In order to proceed further in this direction, we need some results. Let us define

$$
S_{\alpha+\beta}\left(\mathbf{R}^{N}\right)=\inf _{u \in W^{1,2}\left(R^{N}\right) \backslash\{0\}} \frac{\int_{R^{N}}|\nabla u| d z}{\left(\int_{R^{N}}|u|^{\alpha+\beta} d z\right)^{\frac{2}{\alpha+\beta}}}
$$


12 D. C. de Morais Filho, João Marcos do Ó \& M. A. S. Souto

and

$$
\widetilde{S}_{(\alpha, \beta)}\left(\mathbf{R}^{N}\right)=\inf _{(u, v) \in\left\{W^{1,2}\left(\mathbf{R}^{N}\right) \times W^{1,2}\left(\mathbf{R}^{N}\right)\right\} \backslash\{0\}} \frac{\int_{R^{N}}\left(|\nabla u|^{2}+|\nabla v|^{2}\right) d z}{\left(\int_{R^{N}}|u|^{\alpha}|v|^{\beta} d z\right)^{\frac{2}{\alpha+\beta}}} .
$$

Two of the authors together with Alves in [3] proved that

$$
\widetilde{S}_{(\alpha, \beta)}\left(\mathbf{R}^{N}\right)=\left[\left(\frac{\alpha}{\beta}\right)^{\frac{\beta}{\alpha+\beta}}+\left(\frac{\alpha}{\beta}\right)^{\frac{-\alpha}{\alpha+\beta}}\right] S_{\alpha+\beta}\left(\mathbf{R}^{N}\right),
$$

and that if $z_{o}$ realizes $S_{\alpha+\beta}\left(\mathbf{R}^{N}\right)$, then $\left(w_{o}, v_{o}\right)$ realizes $\widetilde{S}_{(\alpha, \beta)}\left(\mathbf{R}^{N}\right)$,

$$
\text { for all } w_{o}=B z_{o} \text { and } v_{o}=C z_{o} \text { with } \frac{B}{C}=\sqrt{\frac{\alpha}{\beta}} \text {. }
$$

In what follows, for $\alpha+\beta=2^{*}$, we shall denote

$$
S_{\alpha+\beta}\left(\mathbf{R}^{N}\right)=S_{*}
$$

and

$$
\widetilde{S}_{(\alpha, \beta)}\left(\mathbf{R}^{N}\right)=\widetilde{S}_{*} .
$$

Remark 2. In [25] it is proved that $S_{*}$ is realized by the oneparameter family of functions

$$
\omega_{\epsilon}(x)=\frac{[N(N-2) \epsilon]^{\frac{N-2}{4}}}{\left(\epsilon+|x|^{2}\right)^{\frac{N-2}{2}}}, \epsilon>0 .
$$

Lemma 5.3. If (5.4) or (5.4) hold then there is a $c$ in (5.8) such that

$$
c<\frac{1}{N} 2\left(\frac{\widetilde{S}_{*}}{2}\right)^{\frac{2}{N}}
$$


Proof. Let $\varphi \in C_{o}^{\infty}\left(\mathbf{R}^{N}\right)$ be a cut-off function with support on the ball $B_{2 R}$, centered at the origin with radius $2 R$, and such that $\varphi \equiv 1$ on $B_{R}$ and $0 \leq \varphi \leq 1$ on $B_{2 R}$. Define $\psi_{\epsilon}(x)=\varphi(x) \omega_{\epsilon}(x)$ and $v_{\epsilon} \equiv \frac{\psi}{\left(\int_{\mathbf{B}_{2 R}} \psi_{\epsilon}^{2^{*}} d z\right)^{\frac{1}{2^{*}}}}$ with

$$
\int_{\mathbf{R}^{N}}\left|v_{\epsilon}^{+}\right|^{2^{*}} d z=1
$$

Let us consider constants $B$ and $C$ such that $\frac{B}{C}=\sqrt{\frac{\alpha}{\beta}}$.

Then for $t>0$ and a fixed $\epsilon>0$ we have

$$
\begin{aligned}
I\left(t B v_{\epsilon}, t C v_{\epsilon}\right)= & \frac{t^{2}}{2}\left[B^{2}\left\|v_{\epsilon}\right\|_{a}^{2}+C^{2}\left\|v_{\epsilon}\right\|_{b}^{2}\right] \\
& -\frac{2 \lambda}{q} B^{\gamma} C^{\delta} t^{q} \int_{\mathbf{B}_{\mathbf{2}}}\left(v_{\epsilon}^{+}\right)^{q}-\frac{2}{2^{*}} t^{2^{*}} B^{\alpha} C^{\beta}
\end{aligned}
$$

The $t$-function in the right hand side of the above equality has a maximum at the point $t_{\epsilon}$ such that

$$
0<t_{\epsilon}<\left\{\frac{1}{2} \frac{B^{2}\left\|v_{\epsilon}\right\|_{a}^{2}+C^{2}\left\|v_{\epsilon}\right\|_{b}^{2}}{B^{\alpha} C^{\beta}}\right\}^{\frac{1}{2^{*}-2}}:=t_{o}^{\frac{1}{2^{*}-2}}
$$

Hence

$$
\begin{gathered}
I\left(t_{\epsilon} B v_{\epsilon}, t_{\epsilon} C v_{\epsilon}\right)=B^{\alpha} C^{\beta}\left[t_{\epsilon}^{2} t_{o}^{2^{*}-2}-\frac{2}{2^{*}} t_{\epsilon}^{2^{*}}\right] \\
-\lambda C_{\epsilon} \int_{\mathbf{B}_{2 R}}\left(v_{\epsilon}^{+}\right)^{q} .
\end{gathered}
$$

Note that the function $t^{2} t_{o}^{2^{*}-2}-\frac{2}{2^{*}} t^{2^{*}}$ is increasing in the interval $\left[0, t_{o}\right)$. This fact together with (5.17) and (5.18) yields that

$$
I\left(t_{\epsilon} B v_{\epsilon}, t_{\epsilon} C v_{\epsilon}\right) \leq \frac{2}{N}\left[\frac{1}{2} \frac{B^{2}\left\|v_{\epsilon}\right\|_{a}^{2}+C^{2}\left\|v_{\epsilon}\right\|_{b}^{2}}{\left(B^{\alpha} C^{\beta}\right)^{\frac{2}{2^{*}}}}\right]^{\frac{N}{2}}-\lambda C_{\epsilon} \int_{\mathbf{B}_{2 R}}\left(v_{\epsilon}^{+}\right)^{q}
$$

and then that

$I\left(t_{\epsilon} B v_{\epsilon}, t_{\epsilon} C v_{\epsilon}\right) \leq \frac{2}{N}\left[\frac{1}{2} \frac{B^{2}+C^{2}}{\left(B^{\alpha} C^{\beta}\right)^{\frac{2}{2^{*}}}}\right]^{\frac{N}{2}}\left[\int_{\mathbf{R}^{N}}\left|\nabla v_{\epsilon}\right|^{2} d z+\int_{\mathbf{B}_{2 R}}(a+b) v_{\epsilon}^{2}\right]^{\frac{N}{2}}$ 
14 D. C. de Morais Filho, João Marcos do Ó É M. A. S. Souto

$$
-\lambda C_{\epsilon} \int_{\mathbf{B}_{2 R}}\left(v_{\epsilon}^{+}\right)^{q}
$$

In [19] it is shown that

$$
\begin{gathered}
{\left[\int_{\mathbf{R}^{N}}\left|\nabla v_{\epsilon}\right|^{2} d z+\int_{\mathbf{B}_{2 R}}(a+b) v_{\epsilon}{ }^{2}\right]^{\frac{N}{2}} \leq S_{*}^{\frac{N}{2}}+O\left(\epsilon^{\frac{N-2}{2}}\right)+} \\
C_{1} \int_{\mathbf{B}_{2 R}}(a+b) v_{\epsilon}^{2}
\end{gathered}
$$

for some constant $C_{1}$, and that if (5.4) or (5.5) hold then

$$
\lim _{\epsilon \rightarrow \infty} \frac{1}{\epsilon^{\frac{N-2}{N}}}\left(C_{1} \int_{\mathbf{B}_{2 R}}(a+b) v_{\epsilon}^{2}-\lambda C_{\epsilon} \int_{\mathbf{B}_{2 R}}\left(v_{\epsilon}^{+}\right)^{q}\right)=-\infty
$$

Using (5.19), (5.13),(5.12), (5.21) and (5.20) for a sufficiently small $\epsilon$, we deduce (5.14).

In order to prove that $u_{o}, v_{o} \neq 0$, one observes that $u_{o}=0$ if and only if $v_{o}=0$. Taking in account the convergence of the sequence $\left(u_{n}, v_{n}\right)$ and (5.11) and (5.14), the rest of the proof follows the same steps of the previous section .

Since $I^{\prime}\left(u_{n}, v_{n}\right)\left(u_{n}^{-}, v_{n}^{-}\right)=\left\|\left(u_{n}^{-}, v_{n}^{-}\right)\right\|_{E}^{2}$, passing to the limit in this equality and using (5.8) we may assume that $u_{o}, v_{o} \geq 0$. A Maximum Principle implies that $u_{o}, v_{o}>0$, as we wanted to prove. The proof of Theorem 5.1 is finished.

\section{ACKNOWLEDGMENTS}

Part of this work was done while the authors was visiting the Departamento de Matemáticas y C. C. of Universidad de Santiago de Chile. They thanks all the faculty and staff of DMCC-USACH for their kind hospitality. The authors also wish to express their gratitude to Professor Claudianor Alves for his valuables comments and suggestions during theelaboration of this work. 


\section{References}

[1] C. O. Alves, Existência de soluções positivas de equações elípticas não-lineares variacionais em $\mathbf{R}^{N}$, Tese de Doutorado, Universidade de Brasília, Brasil, (1995)

[2] C.O. Alves - J. V. Gonçalves - O. H. Miyagaki, Multiple positive solutions for semilinear elliptic equations in $\mathbf{R}^{N}$ involving critical exponents. preprint (1996)

[3] Alves, C.O; D. C. de Morais Filho \& M. A. S. Souto, On a system of elliptic equations with subcritical Sobolev exponent, Proceedings of the 450 Seminário Brasileiro de Análise- FlorianópolisBrasil (1997)

[4] J. G. Azorero - I. P. Alonzo, Existence and nonuniqueness for pLaplacian: nonlinear eigenvalues, Comm. in Partial Differential Equations 12(12), pp. 1389-1430 (1987)

[5] J. G. Azorero - I. P. Alonzo, Problemas elípticos no lineales con exponentes críticos: falta de compacidad,

[6] J. G. Azorero - I. P. Alonzo, Multiplicity of solutions for elliptic problems with critical exponent or with a nonsymmetric term, Transactions of the AMS, Vol. 323, No. 2, Feb, pp. 877-895, (1991).

[7] V. Benci - G. Cerami, Existence of positive solutions of the equations $\Delta u+a(x) u=u^{\frac{N+2}{N-2}}$ in $\mathbf{R}^{N}$, J. Funct. Anal. 88, pp. 90-117, (1990).

[8] H. Berestycki - P. L. Lions, Une methode locale pour l'existence de solutions positives de problèmes semi-lineaires elliptiques dans $\mathbf{R}^{N}$, Journal D'Analyse Mathématique, vol. 38 , pp. 144 - 187, (1980). 
16 D. C. de Morais Filho, João Marcos do Ó \& M. A. S. Souto

[9] H. Brèzis - E. Lieb, A relation between pointwise convergence of functions and convergence functional, Proc. Amer. Math. Cos. 88, pp. 486-490, (1983).

[10] H. Brezis - L. Nirenberg, Some variational problems with lack of compactness, Proc. Symp. Pure Math. (AMS) 45, pp. 165-201, (1986).

[11] H. Brezis - L. Nirenberg, Positive solutions of nonlinear elliptic equations involving critical Sobolev exponent, Comm. Pure and Appl. Math. 36, pp. 437-477, (1983).

[12] D. G. Costa, On a nonlinear elliptic problem, preprint, (1993)

[13] Y. Ding and S. Li, Existence of Solutions for Some Superlinear or Sublinear Elliptic Systems on $\mathbf{R}^{N}$, preprint (1993).

[14] H. Egnell, Semilinear elliptic equations involving critical Sobolev exponents, Arch. Rat. Mech. Anal. 104, pp. 27-56, (1988).

[15] H. Egnell, Existence and nonexistence results for m-Laplace equations involving critical Sobolev exponents, Arch. Rat. Mech. Anal. 104, pp. 57-77, (1988).

[16] H. Egnell, Elliptic boundary value problems with singular coefficients and critical nonlinearities, Indiana Univ. Math. Journal, Vol. 38, No. 2, pp. 235-251, (1989).

[17] Kavian O., Introducion à théorie des points critiques et applications aux problèmes elliptiques, Universitè de Nancy, (1993).

[18] P. L. Lions, Symétrie et compacité dans les espaces de Sobolev, Journal of Functional Analysis 49, pp. 315-334 (1982).

[19] O. Miyagaki, On a class of semilinear elliptic problems in $\mathbf{R}^{N}$ with critical growth, CMS Technical Report \# 94-12, Univ. Winscosin (1994).

[20] J. Mawhin - M. Willem, Critical point theory and Hamiltonian systems. Springer Verlag, Berlin (1989). 
[21] W. Omana and M. Willem, Homoclinic Orbits for a Class of Hamiltonian Systems, Diff. and Int. Eq. 5, pp. 1115-1120.

[22] R. S. Palais, The principle of Symmetric Criticality, Mathematical Physics (1979)

[23] P. H. Rabinowitz, On a Class of nonlinear Schödinger equations, ZAMP 43, (1992).

[24] W. A. Strauss, Existence of solitary waves in higher dimensions, Comm. Math. Phys. 55, pp. 149-162, (1977).

[25] G. Talenti, Best constant in Sobolev inequality, Ann. Math. 110, pp. 353-372 (1976).

Received : May 12, 1999.

Daniel C. de Morais

Marco Aurelio S. Souto

Departamento de Matemática e Estatística

Universidade Federal da Paraíba -UFPB,Campus II

Caixa Postal 10044

58109- 970 Campina Grande

Paraíba

Brasil

and

João Marcos do Ó

CCEN - Departamento de Matemática

Universidade Federal de Paraíba - Campus I

58059.900 João Pessoa

Paraíba

Brasil 